\title{
Axial force prediction based on signals of the elastic wave propagation and artificial neural networks
}

\author{
Piotr Nazarko ${ }^{1, *}$ \\ ${ }^{1}$ University of Technology, Department of Structural Mechanics, Rzeszow, Poland
}

\begin{abstract}
The identification of internal forces is not only important to preserve the structure integrity but also to understand how their certain elements and connections work. Two examples of laboratory test are discussed in this paper. The first is related to an aluminium rod mounted in a stand where compression load was applied. Due to the relaxation phenomenon force prediction becomes even more important in case of compressed bolts. Thus, the second example is related to a bolted flange connection during static tensile test. Four out of six bolts were equipped with washer load cells. Alternatively, selected bolts were equipped with piezoelectric transducers (actuator and sensor) in order to measure signals of elastic waves. It was noted that the load increasing causes changes in the measured signals. Principal components analysis was used for dimensionality reduction of measured signals. The aim of this study is to investigate the use of elastic waves and artificial neural networks for the purpose of the force of identification. Examples of preliminary results have shown that axial forces may be estimated with relatively good accuracy.
\end{abstract}

\section{Introduction}

Measurements of physical quantities describing the state of structural elements are important in many industrial and engineering applications, including civil engineering. Most often they are carried out during trial loads and laboratory tests of prototype solutions to study the behaviour the entire structure and its individual components. The second area are non-destructive tests (NDT) and structural health monitoring (SHM) systems which enhance safety and reliability of structures. Among them, there are several solutions to detect anomalies or damages, classify type of a damage, predict loads or internal forces and to identify material parameters [1-4].

There exists a group of joints where the level of pretension force influences the strength of a slip resistance connection. In this case, pretension force changes over time may become a very important issue for the structure integrity, especially in cyclically-loaded constructions such as bridges, telecommunication towers or wind turbines.

One of the major problems with the use of bolted joints is the precision of tightening method selected to achieve an accurate preload level. Insufficient preload of bolts is a frequent reason of bolted joint failures. There are few main tightening methods used to control the preload of a threaded fastener. Of course, they differ in terms of accuracy. For everyday purposes the clamping force can be approximated by measuring the tightening torque ( $\pm 25 \%$ ), whereas the highest accuracy is achieved using strain gauges or ultrasonic sensing $( \pm 1 \%)$. The force in a single bolt can be estimated using commercial solutions like ultrasonic load monitoring devices. Measurement uncertainty of washer like strain or piezoelectric load cells is about $\pm 10 \%$. Some of them were compared by Fric et al. [5]. Unfortunately, their price can not be possibly neglected and thus in long term research conducted strain gauges are used, either glued onto the bolt or fitted inside the bolt shank.

In this paper a new idea of the force identification is proposed. It takes an advantage of the elastic wave propagation phenomenon. Piezoelectric transducers are used to introduce and measure time signals of elastic waves. Nazarko and Ziemiański [6] have used this idea in the field of SHM and non-destructive damage detection in various materials. Chaki and Bource [7] have studied guided ultrasonic waves to monitor the stress levels in steel strands.

Laboratory tests were performed in two different types of tasks: 1) an aluminium rod under compression, 2) bolts of a flange connection under tension in a static test machine. It was noticed that force changes influence signals measured by sensors. It was reflected also in calculated principal components, which are often used to compress the signals, see Nazarko [8] or Chen et al. [9], where also other signal compression methods are compared. The obtained patterns and data base were then used for the training of Artificial Neural Networks (ANNs). Preliminary obtained results showed that ANNs are able to predict the force in bolts with reasonably well accuracy.

* Corresponding author: pnazarko@prz.edu.pl 


\section{ANNs}

ANNs are widely used in many areas and tasks. The assumption is that ANNs are able to learn an unknown relation between an input and an output data. It typically requires large amounts of data acquired in computational or experimental investigation. In the approach described in this article, standard ANNs were trained to predict axial forces in the investigated elements.

The learning process consists of minimizing the computed error value between the target and the network outputs obtained for successive iterations. Testing and validating is carried out based on the data that the network has never seen before. The ability to produce such a prediction for the training set is called network generalization [10].

The force identification provides information about the predicted value of that force with respect to parameters that are sensitive enough to its changes. The correct selection of these parameters is the most important issue in any identification task. Then, the accuracy of the neural predictor may be obtained by tuning the architecture or different training strategies. For the mentioned task feed forward ANNs are commonly used [10]. They consist of an input (first) layer, usually one or two hidden layers and an output layer. The number of elements in the input and output layers is determined by the size of the learning and testing data sets.

\section{Force prediction}

\subsection{Compression force in an aluminium rod}

The aluminum rod $(12 \mathrm{~mm}$ in diameter, length $70 \mathrm{~cm})$ was placed in a didactic stand (Gunt, WP 120, Fig. 1) and subjected to compression loads. The force values were varying from 100 to $2000 \mathrm{~N}$ with increments of $50 \mathrm{~N}$. Piezoelectric transducers (Noliac CMAP06) were mounted at both ends of the rod. One of them was used to actuate the propagation of elastic waves and the other

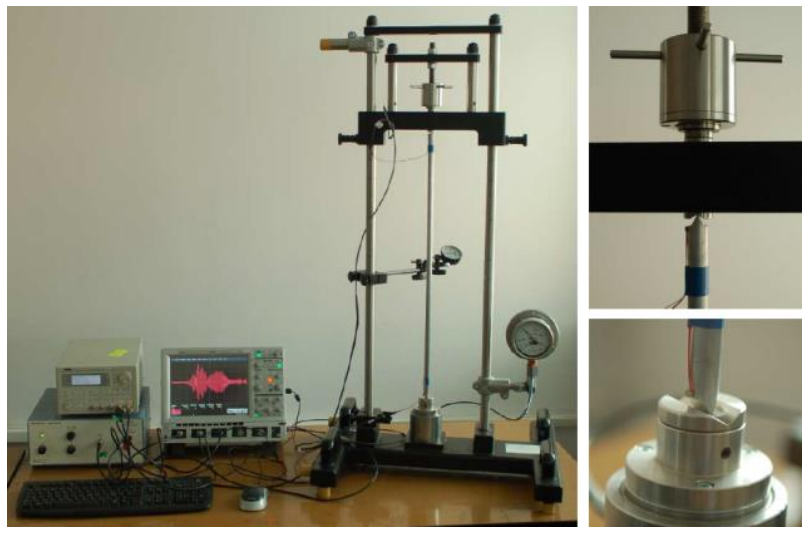

Fig. 1. A laboratory setup and boundary conditions of the studied aluminium rod under compression test

one to capture the answer of the specimen studied. An excitation was defined as 3.5 sine wave modulated by Hanning window with amplitude of $2 \mathrm{~V}$. The received time signals were recorded using a digital oscilloscope with a sampling frequency of $20 \mathrm{MHz}$, see Fig. 1 .

The load was applied by tightening the screw located on the top of the tripod. The magnitude of the force was hydraulically measured and displayed on a force gauge.

An objective of the test was to estimate the amount of compressive forces based on changes in the parameters of registered elastic wave signals. These experiment was carried out in four series in which the specimen was gradually loaded and then unloaded to the initial level of its force. The load ranges and the number of elastic wave time signals recorded were summarized in Table 1 . In this way 248 signals were collected for further investigations.

Table 1. Load ranges and the number of signals recorded in each of the test series

\begin{tabular}{|c|c|c|}
\hline Series & $\begin{array}{c}\text { Load } \\
\text { (with 50 N step) [N] }\end{array}$ & $\begin{array}{c}\text { Number of signals } \\
\text { recorded }\end{array}$ \\
\hline 1 & $100-1000-100$ & 37 \\
\hline 2 & $100-1500-100$ & 57 \\
\hline $3-4$ & $100-2000-100$ & 77 \\
\hline
\end{tabular}

During the rod loading its buckling appeared when the force was bigger than $500 \mathrm{~N}$ and their deflection was increasing with the load magnitude.

\subsubsection{Signal analysis}

The analysis of elastic waves signals was aimed at checking whether parameters of recorded signals change as a result of load changes (in this case compression was varying). Examples of time signals recorded at two selected load values and theirs spectral amplitudes are shown in Fig. 2. One can notice that differences in signals are clearly visible there.

Next, for each signal, their amplitude and time of flight (ToF) observed during the experiment were also determined. In this case it was necessary to increase the sampling rate from the initial value of 5 to $20 \mathrm{MHz}$ to capture changes in the ToF values. The signals were also filtered with bandwidth of 100 and $700 \mathrm{kHz}$. The obtained results are presented in Fig. 3a. It can be noticed that there is a certain relation in the amplitude changes, however, at the same time there are quite significant differences between the results of the first and the other series (2-4). A similar tendency is also visible in Fig. 3b, where signal ToF changes were shown. On this basis, it may be concluded that load increasing was accompanied by increasing in the speed of guided waves. However, the use of ToF requires the further increasing of the signal sampling rate. It can be achieved by the reduction of the signal time base. Unfortunately, it leads also to the shorter signals in time and the smaller amount of information is acquired (i.e. only $0.2 \mathrm{~ms}$ from the signals in Fig. 2a).

For the needs of the performed task, the measured signals have also been transformed into the domain of 

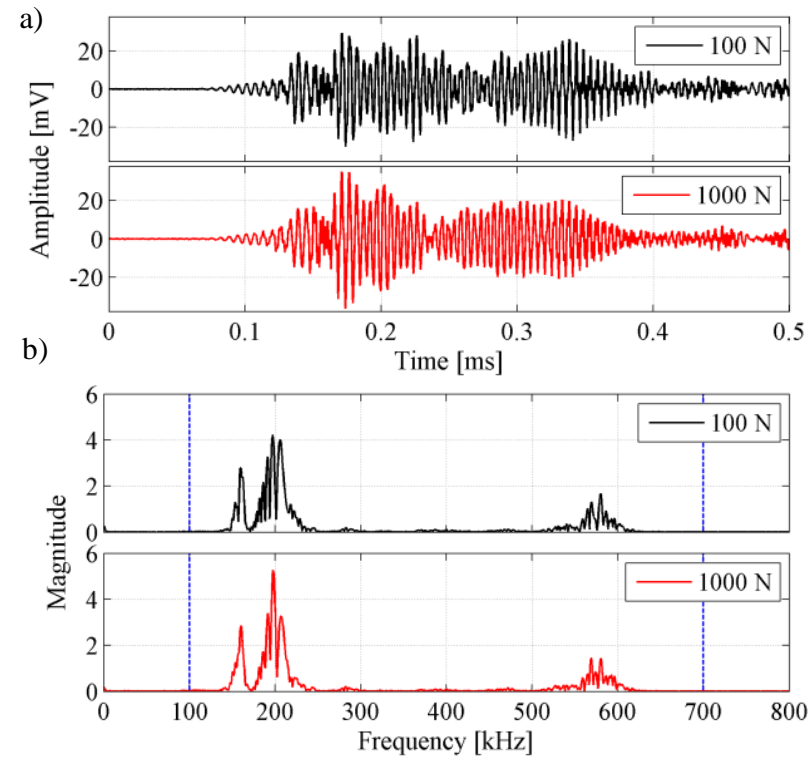

Fig. 2. (a) Exemplary signals recorded at two certain load levels and (b) its spectral amplitudes (first series)

a)
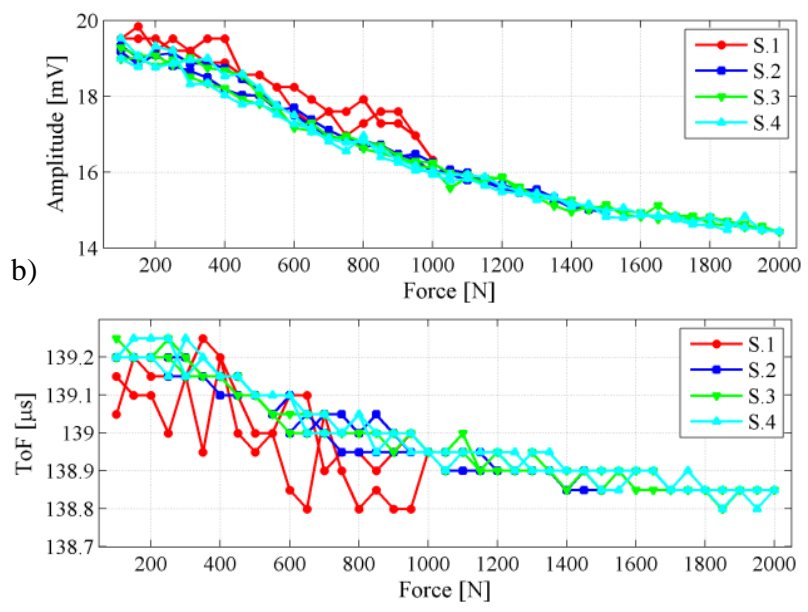

Fig. 3. Variations of selected signal parameters: a) amplitudes, b) phase shift

principal components $[6,8]$. It allows computation of linear transformation

$$
\mathbf{y}=\mathbf{W s}
$$

that maps data from a high dimensional space $\mathbf{s} \subset R_{N}$ to a lower dimensional space $\mathbf{y} \subset R_{K}$ of principal components, without much loss of information. Due to the features of the PCA transformation (matrix multiplication), the elastic wave signals were resampled with the rate $r=10$. As a result, their length was reduced from 10002 to 1001 points. The obtained values of the first three main components are presented in Fig. 4. The representation of multidimen-sional data in $3 \mathrm{D}$ space allows to seea certain relationship between particular series of measurements. Once again, the largest variations of the obtained signal parameters can be seen in the first stage of the loading (estimated as a range up to $400 \mathrm{~N}$ ).
Despite this, it was assumed that the data will be used to train ANN to predict a magnitude of the compressive force.

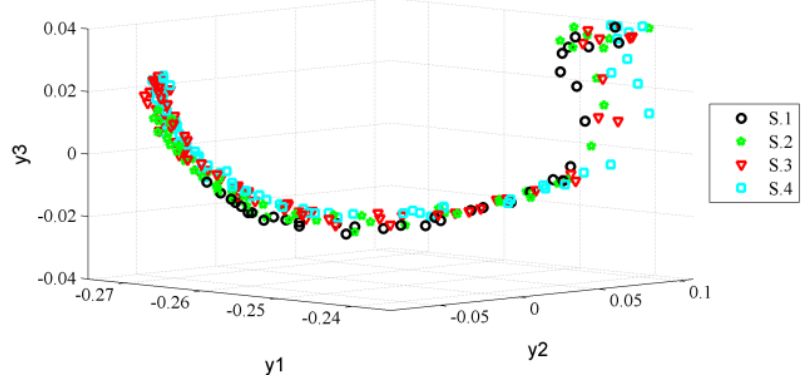

Fig. 4. Projection of multidimensional data into the first three principal components

\subsubsection{Compression force prediction}

The patterns obtained from different measuring series (14) were separated into the following sets: learning (1 and $3)$, validating (2) and testing (4). Only patterns related to the series 1-3 were used during the ANN training, while those from series 4 were used only to simulate the prediction after training process was completed.

The number of 12 principal components was used to build the input vector. In the designed ANN, only one hidden layer was defined and consisted of 5 neurons. This number has been changed within the range of 3 to 8 , however, no significant improvement in the prediction accuracy was found. Thus, the ANN's architecture which was used in this task can be schematically described as 12-5-1.

Exemplary results obtained after ANN training are presented in Fig. 5. It shows a comparison of the obtained values of predicted forces (output) in relation to the expected values of validating patterns (target). The correlation of the this results is very clear. The next graph presents the output force magnitudes in relation to the load history. In addition, the learning, testing and validation sets are highlighted by colors (Fig. 6). In this case the largest obtained validation error did not exceed $80 \mathrm{~N}$. If we refer this value to the maximum force, it gives a relative error of $4 \%$.

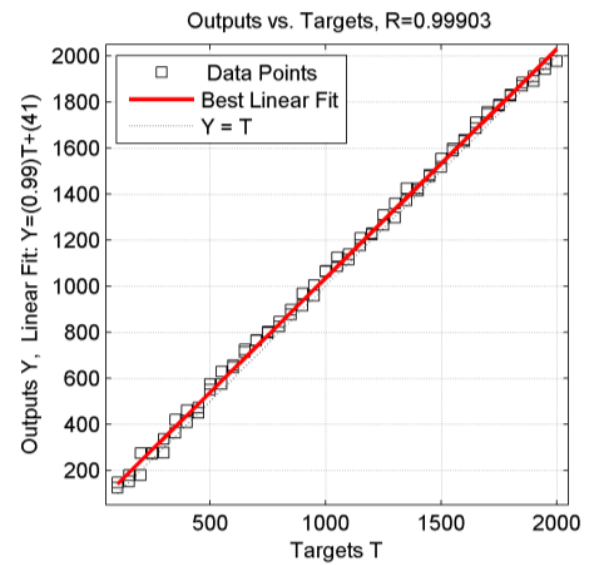

Fig. 5. Exemplary results of the compression force prediction validation set 


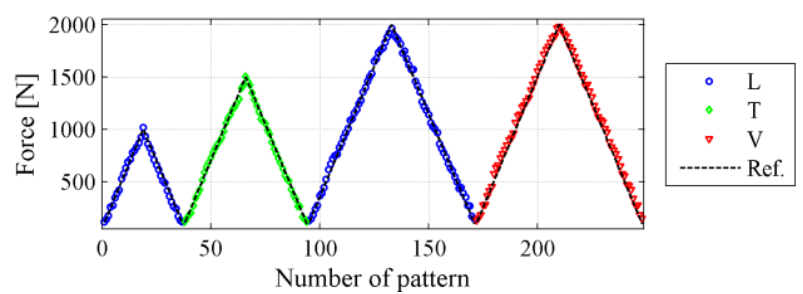

Fig. 6. Force magnitudes estimated by the ANN with respect to the learning, testing and validation sets

\subsection{Force prediction in screws of a flange connection}

\subsubsection{Idea of force monitoring in screws}

The idea proposed in this paper takes advantage of the elastic wave propagation phenomenon. Two piezoelectric transducers (single or placed in two stacks) can be used to excite and to measure signals. The most convenient way of measuring is probably placing the transducers on one side of the screw, but they can use in both a pulse-echo and pitch-catch configurations. It can be expected that even relatively small change in a bolt force will affect the signals measured (its time of flight, amplitude, frequency, etc.), what was confirmed by Kim and Hong [11] and Ding et al. [12]. Parameters describing the force changes can be used for training an efficient diagnosis system which is based on ANNs. The advantage over existing solutions is that it becomes possible to estimate changes in force over time, detection of yielding, fatigue damages and monitoring of the structural integrity.

\subsubsection{Laboratory setup}

The laboratory setup consisted of a signal generator (TTi) where an excitation was defined in the form of 2.5 sine wave modulated by Hanning window. Operational frequency was set to $52 \mathrm{kHz}$. Then the signal was amplified and split to actuators and synchronization channels. Two digital oscilloscopes (LeCroy) where used to store signals received from all the sensors. Piezoelectric transducers (Noliac CMAP6) were mounted on the bolt's head (excitation) and at the end of its shank. The sensor wax used enables trouble-free recovery of all sensors while their cables were fixed in single points with weak adhesive which holds them during test and allows a non-invasive removal.

During initial tests the bolt was equipped with two transducers on its head and one on the end. In this case two measurements scenarios were studied: one related to impulse-echo and the second to pitch-catch approach. It was decided at this stage of investigation that only pitchcatch signals would be used for the purpose of training the diagnosis system.

\subsubsection{Single bolt in a static test machine}

A single bolt M16 class 8.8 and $125 \mathrm{~mm}$ length was placed in the static test machine. It was decided that loads will be applied in four cycles with increasing force levels up to $25,50,75$ and $100 \mathrm{kN}$. The force, in this case, was related with load history read out from the test machine. The set of these values was then used as the output vector during the process of ANNs training.

A total of 602 signals were recorded at intervals of $5 \mathrm{~s}$. Examples of signals obtained at selected load stages were shown in Fig. 7. Amplitude for the selected signals changes are clearly visible there. All the recorded signals were then processed using PCA. Projection of two first principal components is shown in Fig. 8. These values, however, do not indicate a simple dependence on load changes that would allow direct prediction of forces. Therefore, principal components were used to train an

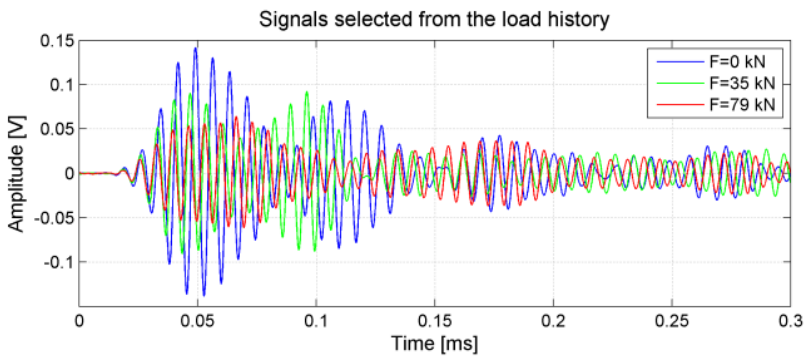

ANN for the purpose of force identification.

Fig. 7. Signals selected from the load history of the single bolt under cyclic load test

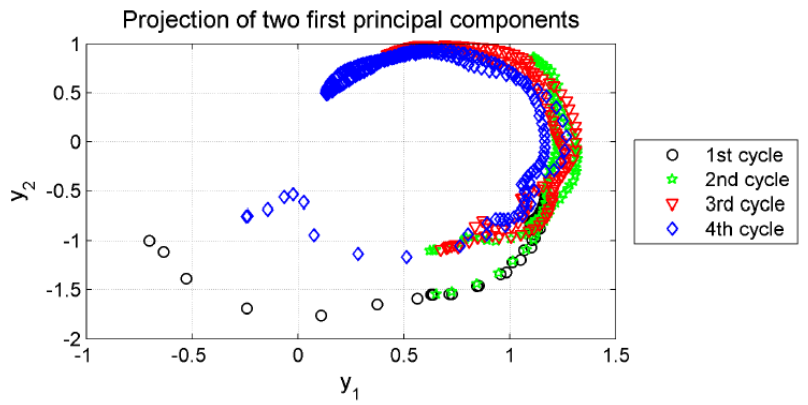

Fig. 8. Projection of two first principal components calculated from elastic wave signals recorded in the single bolt under cyclic load test

Patterns for testing and validation were selected with constant distribution. It means that every third signal was chosen for testing, one third of them was used for validation, while the others patterns have formed learning set. One of the best obtained ANNs training results were shown in Fig. 9. A mean testing error was equal to 1.88 $\mathrm{kN}$ (less than 2\%) with standard deviation of $1.64 \mathrm{kN}$.

\subsubsection{A flange connection under static tests}

An investigated flange connection (Fig. 10) consisted of six bolts M16 class 5.8. Only four of them were equipped with a washer strain load cells (bolts no. 1,2, 4 and 5, see Fig. 10). The other two had spacers that imitate the force sensors. The forces obtained during static tests defined an output vector for ANNs training. It can be seen in Fig. 11 that forces in certain bolts are not the same. This may be related to geometric imperfections of the connection (nonsymmetric) or measurement uncertainty (this kind of sensors is sensitive to the washers hardness, a screw may touch the edge of the hole). 


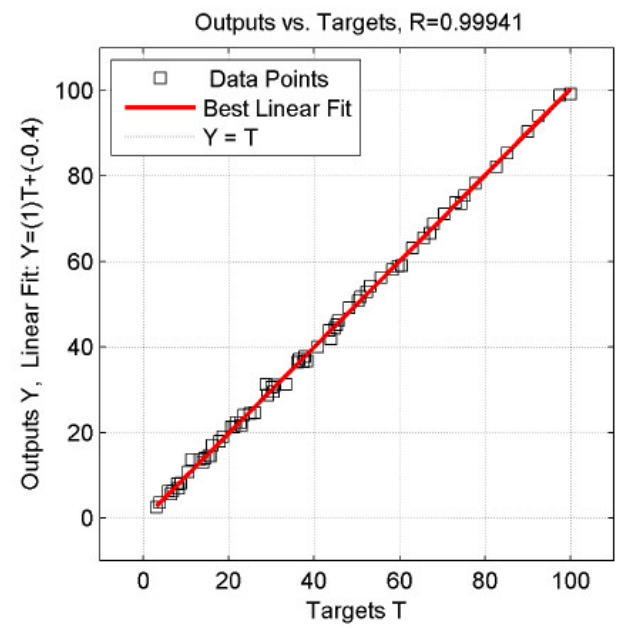

Fig. 9. Results of ANNs training based on cyclic loads of a single bolt (constant patterns distribution): validation error
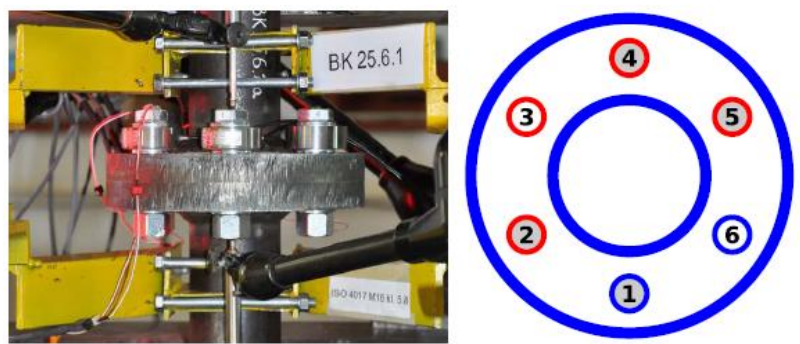

Fig. 10. A flange connection under static tests and a scheme of screw's numbers

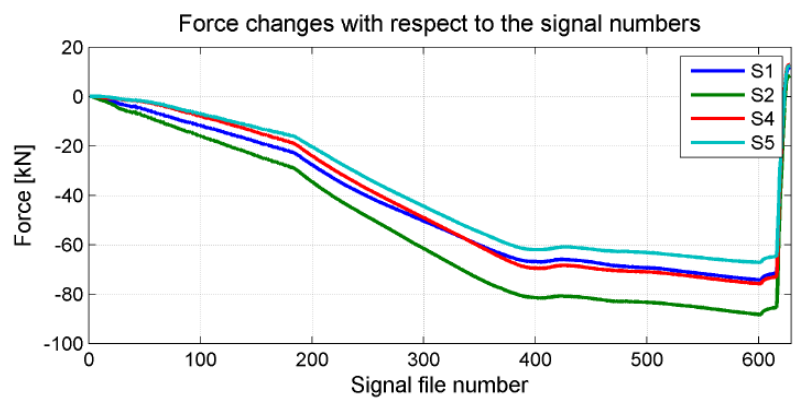

Fig. 11. Force changes measured in bolts of a flange connection under static tests

Four bolts were equipped also with piezoelectric transducers, but their numbers were slightly different, i.e. 2, 3, 4 and 5. Therefore the bolts no 3, which were not equipped with the washer load cell, may be used for the purpose of unknown force prediction based on recorded elastic wave signals and trained ANNs with data from the other bolts.

Elastic wave signals comparison at the initial stage shows that they are not exactly the same. Even after their normalization to the range \pm 0.9 , the differences in the signals are well visible (Fig. 11). Principal components calculated also clearly differ between the individual bolts (Fig. 12). There may be several reasons in this case: piezoelectric transducers are not placed exactly at the same position, the thickness of the wax layer is not the same, the signals are affected by the closest components of the connection (washers, brackets on the pipe, weld thickness, etc.). Despite these differences, an attempt was made to train ANNs for the purpose of force prediction.

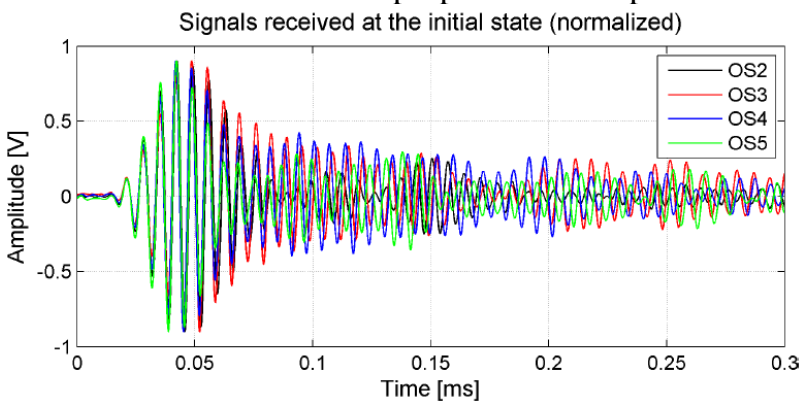

Fig. 12. Time signals of elastic waves measured at the initial state in four bolts investigated

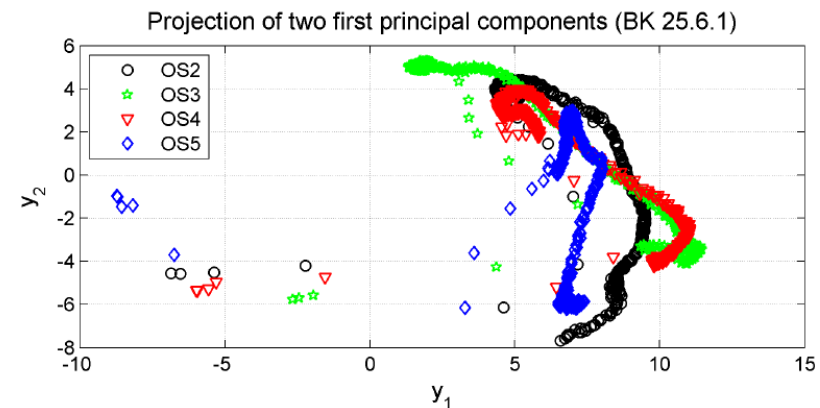

Fig. 13. Projection of two first principal components calculated for all signals received (four bolts and full load history).

First, it was assumed that data from all bolts $(2,4,5)$ would be used for the purpose of ANNs training, assuming a constant distribution of patterns for testing and validation. The obtained results are very promising, what is shown in Fig. 14. The predicted force changes are marked there by points $\mathrm{Ru}, \mathrm{Rt}, \mathrm{Rv}$ with respect to the relation observed during the experiment (comp. Fig. 11 and 14). However, if patterns from the bolts no. 3 were provided to the ANN trained, its generalization ability can not be accepted. It was expected that the predicted force values would be on the same level like the others, while the obtained results are quite unpredictable in the initial phase (see "Rp-" path in Fig. 14).

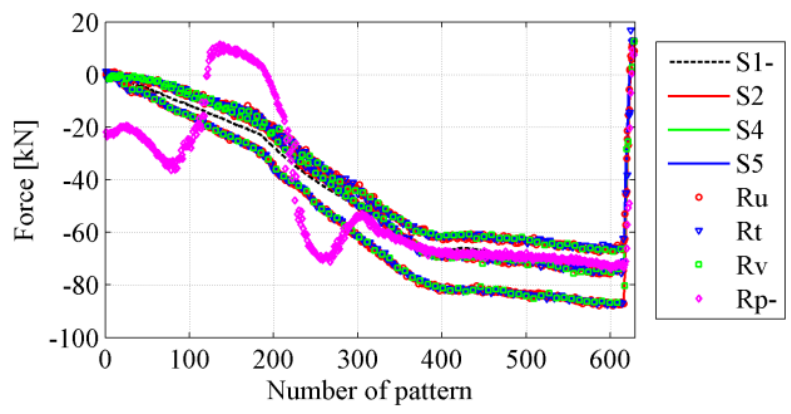

Fig. 14. Force prediction based on the validation set

\section{Conclusions and final remarks}

The obtained results of preliminary tests have showed that ANNs are able to find the relation between the changes in signals and force variations. In case of the aluminium rod under compression loaded up to $2000 \mathrm{~N}$, 
the largest prediction errors did not exceed $80 \mathrm{~N}$. It gives the accuracy of $96 \%$ if compared to the load range.

A more detailed analysis of amplitude and phase changes requires the higher signal resolution where sampling frequency is more than $20 \mathrm{MHz}$.

In the case of the force prediction in bolts of the flange connection, the accuracy was even more than $98 \%$, when testing and validation patterns were selected with constant distribution. It seems, however, that the principal components do not contain information suitable enough for precise prediction of axial forces in bolts that were not included into the training patterns database (even in case of the single connection where environmental conditions were the same).

The signals measured showed significant differences and therefore, at this stage of the research, the trained ANNs were not able to generalize data and to predict the unknown forces with acceptable accuracy.

In the future work the other signal parameters will be studied (ToF, signal amplitudes, wavelet coefficients, etc.) in order to improve the accuracy of the force prediction. The set of training patterns should be also extended with data related to experimental data performed on the wider group of bolts in order to find the cause of the difference observed in the measured signals of elastic waves.

\section{References}

1. M. Sale, P. Rizzo, A. Marzani, MS\&SP, 25 (2011)

2. M. Słoński, Bayesian Machine Learning in Analysis of Selected Identification Problems in Mechanics of Materials and Structures (2014).

3. L. Yu, V. Giurgiutiu, Smart Struc. and Syst., 1 (2005)

4. A. Żak, M. Radzieński, M. Krawczuk, W. Ostachowicz, SMS, 21 (2012)

5. N. Fric, D. Budjevac, Z. Miskovic, M. Veljkovic, Z. Markovic, J. Dobric, 8th Intern. Conf. on Adv. in Steel Struct., Lisbon, Portugal, 1-10 (2015)

6. P. Nazarko, L. Ziemiański, EFA, 69 (2016).

7. S. Chaki, G. Bourse, Ultr., 49 (2009)

8. P. Nazarko, IPSE, 21 (2013).

9. R. Chen, S. Chen, Y. Lixia, J. Wang, X.,Xu, T. Luo, Neurocomp., 219 (2017)

10. Z. Waszczyszyn, L. Ziemiański, In: Z. Mróz, G.E. Stavroulakis (eds) Parameter Identification of Materials and Structures, 469 (CISM Courses and Lectures, Springer, Vienna, 2005).

11. N. Kim, M. Hong, NDT\&E Intern., 42 (2009)

12. X. Ding, X.Wu, Y. Wang, Ultr., 54 (2014) 